\title{
FREQUENCY CONVERSION OF COHERENT IMAGES ON INTRACAVITY MULTIWAVE MIXING
}

Omar Ormachea, Oleg G. Romanov and Alexei L. Tolstik

\begin{abstract}
The schemes for recording and reading of dynamic holograms in conditions of nondegenerate four- and six-wave mixing in a nonlinear Fabry-Perot interferometer have been analyzed theoretically. It has been demonstrated that there is a possibility for a considerable improvement in the diffraction efficiency and angular selectivity of dynamic gratings in the interferometer compared to the off-cavity interaction. A method for the frequency conversion of coherent images with simultaneous phase conjugation has been realized experimentally.
\end{abstract}

Keywords: Dynamic Holography, Frequency Conversion of Images, Nondegenerate Multiwave Mixing, Nonlinear Interferometer.. 